\title{
1
}

\section{Antennas and Antenna Arrays}

\subsection{Introduction}

Antennas have become ubiquitous devices and occupy a salient position in wireless systems. Radio and TV as well as satellite and new generation mobile communications have experienced the largest growth among industry systems. The global wireless market continues to grow at breakneck speed and the strongest economic and social impact nowadays comes from cellular telephony, personal communications and satellite navigation systems. All of the above systems have served as motivation for engineers to incorporate elegant antennas into handy and portable systems.

Many textbooks provide in-depth resources on antennas. Especially on antenna arrays, there are digests, studies and books containing extensive data and techniques. In the references given herewith [1-26], there are some of the best-known and most highly recommended books.

A device able to receive or transmit electromagnetic energy is called an 'antenna'. As seen in [6], the antenna plays the role of a transitional structure between free space and a guiding device. An antenna consists of one or more elements. A single-element antenna is usually not enough to achieve technical needs. That happens because its performance is limited. A set of discrete elements, which constitute an antenna array, offers the solution to the transmission and/or reception of electromagnetic energy. The geometry and the type of elements characterize an antenna array. For simplicity, implementation and fabrication reasons, the elements are chosen in such a way so as to be identical and parallel. For the same reasons, uniformly spaced linear arrays are mostly encountered in practice.

In the following paragraphs, the properties of various antenna arrays will be presented.

\subsection{Antenna Array Factor}

The radiation characteristics of antennas have mostly to do with the far field (Fraunhofer) region. In this region, the field expression is a multiplication of two parts. One part contains the distance $r$ dependence of the observation point (receiver location) and the other contains its spherical coordinate angles $\theta$ and $\phi$ dependence. The angular distribution

Orthogonal Methods for Array Synthesis: Theory and the ORAMA Computer Tool John N. Sahalos 


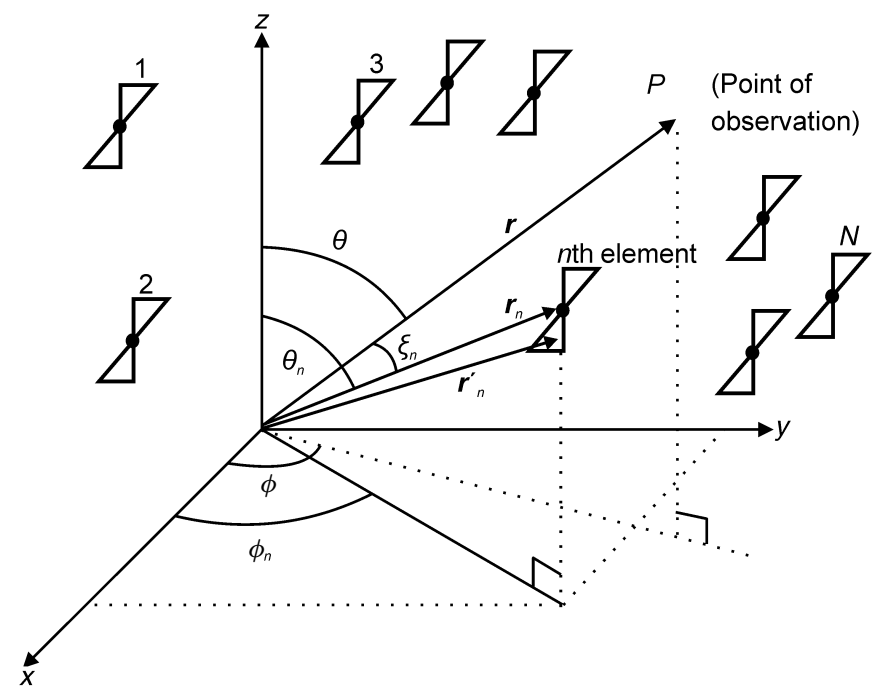

Figure 1.1 Geometry of an antenna array with $N$ identical and parallel elements

of the field is independent of the distance $r$. For a typical antenna element (see Fig. 1.1), the far electric field is

$$
\boldsymbol{E}_{n}(r) \cong-j \omega \mu \frac{\mathrm{e}^{-j \beta r}}{4 \pi r} \boldsymbol{f}_{n}(\theta, \phi)
$$

The angular-dependent vector $f_{n}(\theta, \phi)$ gives the directional characteristics of the $n$th element electric field [11]:

$$
\boldsymbol{f}_{n}(\theta, \phi)=(\hat{\theta} \hat{\theta}+\hat{\phi} \hat{\phi}) \cdot \int_{\text {element }} \boldsymbol{J}_{n}\left(r_{n}^{\prime}\right) e^{j \beta \hat{\boldsymbol{r}} \cdot\left(\boldsymbol{r}_{n}-\boldsymbol{r}_{n}^{\prime}\right)} d v^{\prime}
$$

where

$J_{n}\left(r_{n}^{\prime}\right)=$ electric current density of the $n$th element

$r_{n}^{\prime}=$ distance of a source point from the origin

$r=$ distance of the observation point from the origin

$\beta=\frac{2 \pi}{\lambda}$ the free space wave number

$\omega=$ the angular frequency and

$\mu=$ the magnetic permeability of the space

The total electric field of an $N$ element antenna array is

$$
\boldsymbol{E}(r)=\sum_{n=1}^{N} \boldsymbol{E}_{n}(r)
$$

Moreover, the total magnetic field is [6],

$$
\boldsymbol{H}(r)=\frac{1}{\eta} \hat{\boldsymbol{r}} \times \boldsymbol{E}(r)
$$

where $\eta=\sqrt{\mu / \epsilon}(\epsilon$ is the electric permeability of the space). 
For identical and identically oriented elements, the current distribution of each element is approximately the same except for a constant complex multiplier. In $(1-1), f_{n}(\theta, \phi)$ can be expressed as

$$
\boldsymbol{f}_{n}(\theta, \phi)=I_{n} \boldsymbol{f}(\theta, \phi)
$$

$\boldsymbol{f}(\theta, \phi)$ is called the 'pattern function' of the element and $I_{n}$ is the complex excitation of the $n$th element of the array.

(1-1), (1-2) and (1-5) are combined and give

$$
\boldsymbol{E}(r)=-j \omega \mu \frac{e^{-j \beta r}}{4 \pi r} \boldsymbol{f}(\theta, \phi) \sum_{n=1}^{N} I_{n} e^{j \beta r_{n} \cos \xi_{n}}
$$

$\left(r_{n}, \theta_{n}, \phi_{n}\right)$ are the spherical coordinates of a convenient reference point of the $n$th element and $\cos \xi_{n}=\sin \theta \sin \theta_{n} \cos \left(\phi-\phi_{n}\right)+\cos \theta \cos \theta_{n}$.

The last term of (1-6) is expressed separately as

$$
A F(\theta, \phi)=\sum_{n=1}^{N} I_{n} e^{j \beta r_{n} \cos \xi_{n}}
$$

$A F(\theta, \phi)$ is called the 'array factor'. This factor is actually the array pattern of $N$ isotropic point sources positioned at the reference points of the elements of the original array.

From (1-6) and (1-7), we have the following:

$$
\boldsymbol{E}(r)=-j \omega \mu \frac{e^{-j \beta r}}{4 \pi r} f(\theta, \phi) A F(\theta, \phi)
$$

Expression (1-8) states the following pattern multiplication principle: An array consisting of identical and identically oriented elements has a pattern, which can be expressed as the product of the element pattern and the array factor.

An antenna engineer has to make an anticipatory and compatible choice of elements according to technical requirements. Once the element pattern is derived, the design effort is mainly directed at the array factor.

\subsection{Elements and Array Types}

Element types of antenna arrays are delineated in the literature [1-14]. Dipoles, monopoles, loops, slots, microstrip patches and horns are the most common types of array elements. Recent studies and innovations have resulted in new types of elements. Some of them are the monolithic, the superconducting, the active and the electronically and functionally small antenna elements.

In parallel with the development of elements, antenna arrays have experienced a tremendous growth. Their list starts with the linear broadside and end-fire arrays, the planar, the circular, and the conformal, and goes up to the adaptive arrays. Moreover, flat plate slot arrays, digital beam forming, dichroic, slotted and fractal arrays are some of the recent types.

It was mentioned previously that antenna analysis and synthesis focuses mostly on the array factor. Consequently, in the following paragraphs we devote the analysis mainly to this factor. 


\subsection{Antenna Parameters and Indices}

In many cases, it is necessary to characterize the performance of antennas by referring to specific parameters and indices. Most of these parameters and indices have been defined by the committees of the institutions in charge (IEEE, ETSI etc.) and will be presented in this paragraph.

It is well known that antennas have to do with applications of time varying fields. Sources with $e^{j \omega t}$ time variation produce fields that also vary in the same way. In the literature $[6,22]$, the above fields are called 'time-harmonic fields'. If a signal with certain bandwidth is present in an antenna, the Fourier transform can derive the time varying forms of the electromagnetic quantities. The procedure is analogous to that of solving electric circuit problems. In this book, time-harmonic fields with a proper choice of working frequency/ies are assumed.

\subsubsection{Radiated Power}

The time average power density, which is the average Poynting vector, can be written in the following form:

$$
\boldsymbol{S}=\frac{1}{2} \operatorname{Re}\left[\boldsymbol{E} \times \boldsymbol{H}^{*}\right]
$$

The $1 / 2$ in (1-9) appears because the electric and magnetic fields represent the peak values.

The radiated power $P_{r}$, through a closed surface $S$ surrounding the antenna, can be obtained by integrating the normal component of the average Poynting vector over the entire surface. On the basis of the above definition we have

$$
P_{r}=\oiint_{s} \boldsymbol{S} \cdot d \boldsymbol{s}=\frac{1}{2} \oiint_{s} \operatorname{Re}\left[\boldsymbol{E} \times \boldsymbol{H}^{*}\right] \cdot d \boldsymbol{s}
$$

The surrounding surface can be of arbitrary shape, and without losing generality a sphere is used.

\subsubsection{Radiation Intensity}

Radiation intensity in a given direction is the power radiated per unit solid angle in the above-mentioned direction. It is expressed in watts per unit solid angle and is related to the far field of the antenna. In a spherical coordinate system $(r, \theta, \phi)$, radiation intensity is defined as

$$
U(\theta, \phi)=r^{2} \boldsymbol{S} \cdot \hat{\boldsymbol{r}}
$$

where $r$ denotes the distance between the antenna and the observation point and $\hat{\boldsymbol{r}}$ is the corresponding radial unit vector.

$U(\theta, \phi)$ can be expressed only by the electric or the magnetic far-zone field:

$$
U(\theta, \phi)=\frac{r^{2}}{2 \eta}|\boldsymbol{E}(r, \theta, \phi)|^{2}=\eta \frac{r^{2}}{2}|\boldsymbol{H}(r, \theta, \phi)|^{2}
$$

where $\eta$ is the intrinsic impedance of the medium. (For the free space $\eta=120 \pi$ Ohms). 
The total power $P_{r}$ radiated is obtained by taking the integral of the radiation intensity over all angles around the antenna. Thus, $P_{r}$ is

$$
P_{r}=\oiint U d \Omega=\int_{0}^{2 \pi} \int_{0}^{\pi} U(\theta, \phi) \sin \theta d \theta d \phi
$$

Another parameter related to $U(\theta, \phi)$ is the average radiation intensity, defined as the radiation intensity of an isotropic source radiating the same power as that of the actual antenna. Thus,

$$
U_{\text {ave }}=\frac{1}{4 \pi} \oiint_{\Omega} U d \Omega=\frac{P_{r}}{4 \pi}
$$

where $d \Omega$ is the element of solid angle.

It is obvious that an isotropic source (antenna) is not realizable; however, it is often used as a reference element for many antennas.

\subsubsection{Directivity}

The directivity of an antenna in a given direction is defined as the ratio of the radiation intensity in the above-mentioned direction to the average radiation intensity. If the direction is not specified, then it is implied in the direction of maximum radiation. By using (1-12) and (1-13), directivity $D$ is

$$
D=\frac{U}{U_{\text {ave }}}
$$

Directivity can be more conveniently expressed by

$$
D=4 \pi \frac{U}{P_{r}}
$$

Maximum directivity is given by

$$
D_{\max }=D_{o}=4 \pi \frac{U_{\max }}{P_{r}}
$$

The directivity of an isotropic source is unity. All other sources have maximum directivity greater than unity.

\subsubsection{Antenna Efficiency}

It is well known that only one part of the input power at the input terminals of an antenna is radiated. Various reasons, like the mismatch between the transmission line and the antenna or conduction and dielectric losses of the antenna itself, reduce the power radiated.

The total efficiency of an antenna can be expressed by

$$
e_{o}=e_{r} e_{c} e_{d}
$$

where $e_{o}$ is the total efficiency, $e_{r}$ is the mismatch efficiency $=\left(1-|\Gamma|^{2}\right), e_{c}$ is the conduction efficiency and $e_{d}$ is the dielectric efficiency. $\Gamma$ is the voltage reflection 
coefficient at the input of the antenna terminals expressed by

$$
\Gamma=\frac{Z_{i n}-Z_{o}}{Z_{i n}+Z_{o}}
$$

where $Z_{i n}$ is the antenna input impedance and $Z_{o}$ is the characteristic impedance of the transmission line.

(1-18) is expressed in an alternative form as

$$
e_{o}=e_{c d} e_{r}=e_{c d}\left(1-|\Gamma|^{2}\right)
$$

$e_{c d}$ is the radiation efficiency that can be determined experimentally or, if possible, numerically.

\subsubsection{Gain}

The performance of an antenna can also be described by the gain. The gain is related to directivity. It is an index that takes the directional properties and the efficiency of the antenna into account. The gain $G(\theta, \phi)$ is defined by

$$
G(\theta, \phi)=\frac{\text { radiation intensity for the } \operatorname{direction}(\theta, \phi)}{\frac{1}{4 \pi}(\text { power input to the antenna })}
$$

When the direction is not specified, the gain is taken in the direction of maximum radiation. Expression (1-21) counts the losses of the antenna itself and can be written as

$$
G(\theta, \phi)=e_{c d} \frac{U(\theta, \phi)}{\frac{1}{4 \pi} P_{r}}=e_{c d} D
$$

If one takes the mismatch losses into account, then the absolute gain $G_{a b s}$ is introduced [6]. $G_{a b s}$ is expressed by

$$
G_{a b s}=e_{o} D
$$

Finally, maximum absolute gain $G_{o_{a b s}}$ is related to maximum directivity $D_{o}$. It is

$$
G_{o_{a b s}}=e_{o} D_{o}
$$

The above indices are given in terms of decibels instead of dimensionless quantities. The relation of an index $X$ in $\mathrm{dB}$ and its corresponding dimensionless value is

$$
X[\mathrm{~dB}]=10 \log X[\text { dimensionless }]
$$

\subsubsection{Antenna Patterns}

One of the main characteristics of an antenna is its radiation pattern. It presents graphically the radiation properties and can be measured by moving a probe antenna around the antenna under test at a constant distance in the far field (see Fig. 1.2a). The response, as a function of the angular coordinates, constitutes the radiation pattern. Depending on 


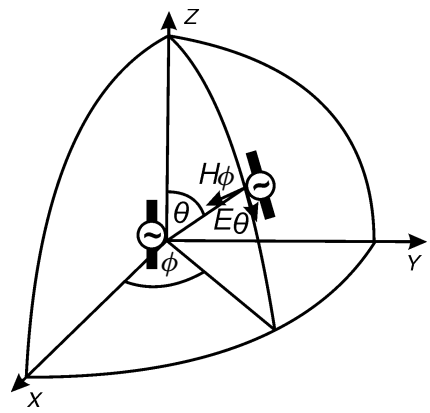

(a)

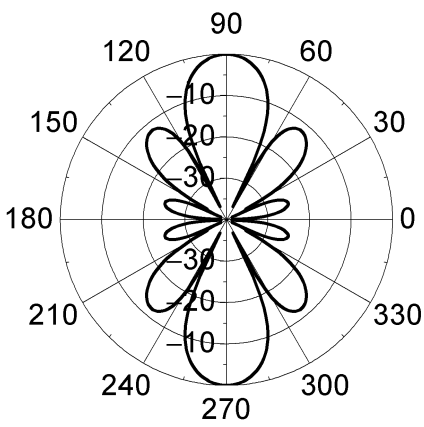

(c)

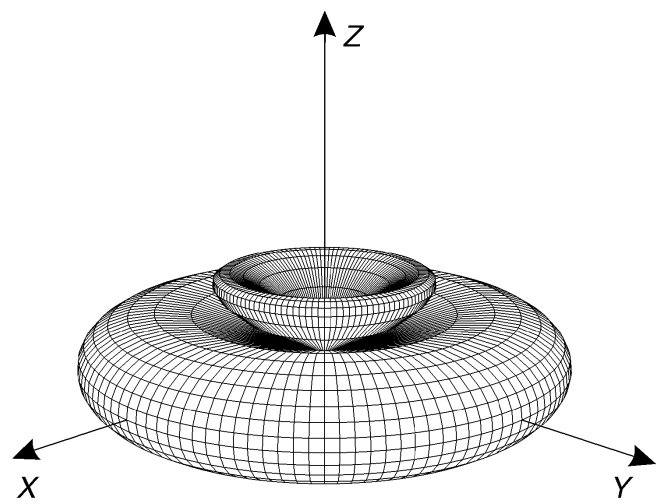

(b)

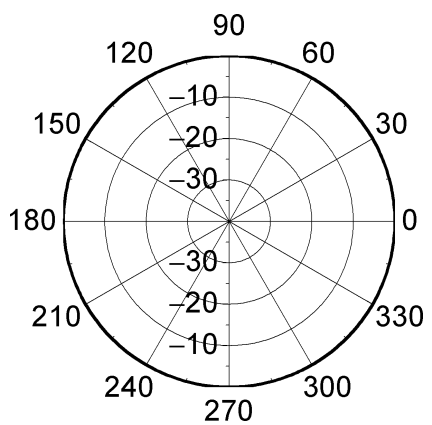

(d)

Figure 1.2 Radiation pattern of a linear array of three vertical collinear electric $\lambda / 2$ dipoles in $\mathrm{d} / \lambda=0.75$ (a) Pattern measurement scheme, (b) Three-dimensional plot, (c) E-plane radiation pattern, (d) H-plane radiation pattern

probe type and orientation, the appropriate component of an electric or a magnetic field can be measured. If a probe is moved over the spherical surface, its terminal voltage will present the 3-D radiation pattern. A pattern taken on one plane is known as a "plane pattern'. The pattern that contains the electric field vector is the E-plane pattern while the pattern that contains the magnetic field vector is the H-plane. The above two are referred to as the 'principal plane patterns'. As an example, Fig. 1.2b presents the 3-D radiation pattern of a uniform linear array of three collinear vertical electric dipoles with equal mutual distance of $0.75 \lambda$. Figures $1.2 \mathrm{c}$ and $\mathrm{d}$ show the $\mathrm{E}-$ and H-plane patterns of the array.

A plane pattern can be depicted as a polar or as a rectangular plot. The units of the patterns are either linear (Fig. 1.3a) or logarithmic (dB) (Fig. 1.3b). The lobe in the direction of maximum radiation is called the 'main lobe' or the 'main beam'. Any lobe of the pattern other than the main lobe is a 'minor lobe'. Usually, a minor lobe is also called a 'side lobe'. A side lobe in a pattern is, in general, any lobe other than that of the intended one. Since the intended lobe is usually the main lobe, it is obvious that minor 


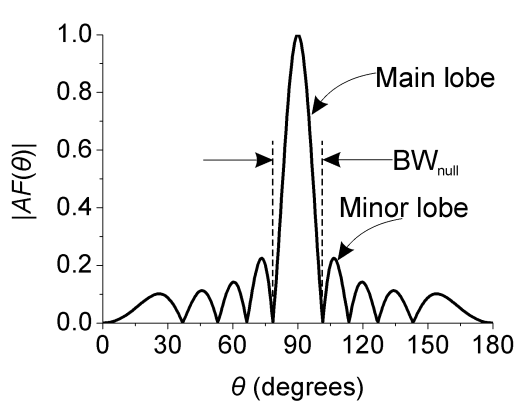

(a)

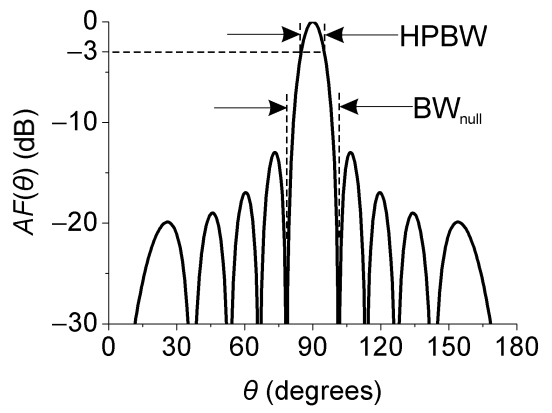

(b)

Figure 1.3 Radiation patterns in (a) linear and (b) logarithmic scale

and side lobes are the same. A measure of the characteristics of the pattern is given by certain quantities:

1. The half-power (or $3 \mathrm{~dB}$ power) beam width (HPBW) is the angular width between the angular points half-power $(3 \mathrm{~dB})$ below that of the main-beam maximum of the antenna (see Fig. 1.3b).

2. The first null beam width $\left(\mathrm{BW}_{\text {null }}\right)$ is defined as the angular width between the first zero crossing of either side of the main-beam maximum of the antenna (see Figs. 1.3a and $b$ ).

3. The bandwidth $\Delta f$ of an antenna is defined by the frequency limits at which the maximum gain is reduced to its half value $(3 \mathrm{~dB})$. The fractional bandwidth is given by $\Delta f / f . f$ is the mean operating frequency of the antenna.

4. The side lobe level (SLL) is the ratio of the pattern value of a side lobe peak to the corresponding value of the main lobe. Usually, SLL in an antenna is defined as the largest side lobe level for the whole pattern. A special case of the inverse SLL is the front to back ratio $(\mathrm{F} / \mathrm{B})$. This is the ratio of the pattern value in the main lobe maximum to the corresponding value in the direction of 180 degrees from it. If there is a minor lobe in the back direction, this is called a 'back lobe' (see Fig. 1.4a).

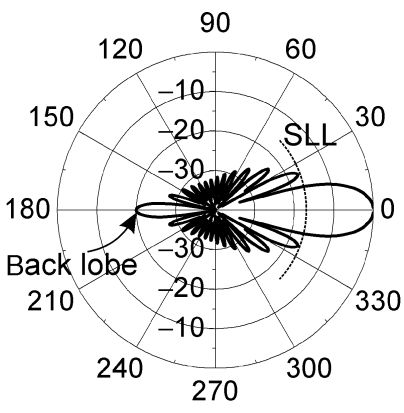

(a)

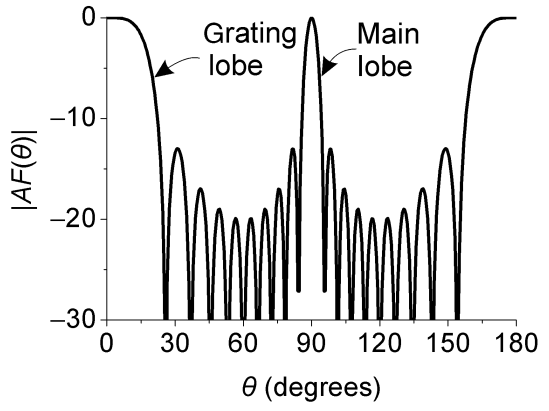

(b)

Figure 1.4 (a) Polar plot of an antenna pattern with $\mathrm{SLL}=-17.24 \mathrm{~dB}$ and $\mathrm{F} / \mathrm{B}=20 \mathrm{~dB}$, (b) Rectangular plot of an antenna with grating lobes 
5. The grating lobe is any maximum equal to the maximum of the main lobe of the pattern. One or more grating lobes are formed in antenna arrays when the spacing between the elements is more than $\lambda$ (see Fig. 1.4b).

Depending on its radiation pattern, an antenna is called 'broadside', 'end-fire' or 'intermediate'. A broadside antenna is an antenna for which the direction of the main lobe maximum is normal to the plane containing the antenna. If the above direction is within the plane, then the antenna is an end-fire antenna. An antenna is intermediate if it is neither broadside nor end-fire. Figures $1.5 \mathrm{a}, \mathrm{b}$ and $\mathrm{c}$ represent the pattern of the three types of antennas. The beam of the antenna in Fig. 1.5a is known as a 'fan beam'. The single lobe of the antenna in Fig. 1.5b is called a 'pencil beam'.

It is noticed that, owing to reciprocity, the radiation pattern of an antenna in the transmitting mode is identical to that in the receiving mode. The reciprocity theorem is well known from circuit analysis [27]. It states: If a constant current (voltage) source is placed in one branch of a reciprocal network, and a voltage (current) reaction is measured in another, then interchanging the locations between the source and the branch of measurement, we have unchanged measurement results. A network is reciprocal when it is composed of linear, bilateral elements. In antennas, the materials of the construction, the transmission line and the medium of wave propagation must be linear. Nonlinear devices (diodes, transistors) make the antenna nonreciprocal. In electromagnetics, the reciprocity

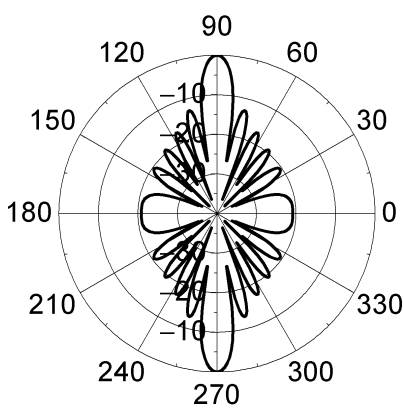

(a)

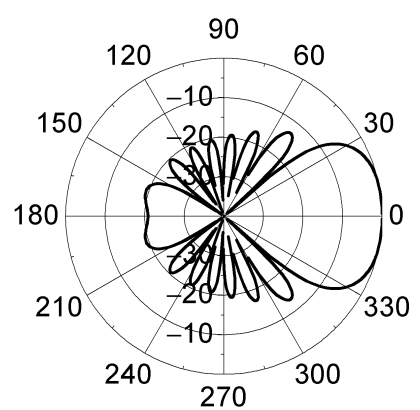

(b)

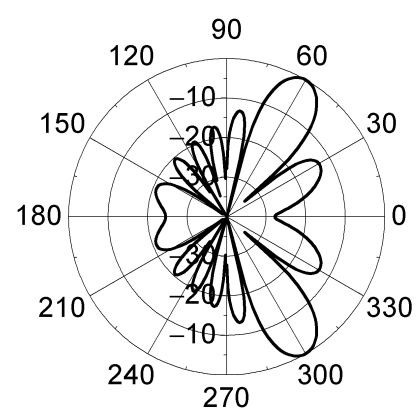

(c)

Figure 1.5 Radiation pattern of (a) A broadside array (fan beam), (b) An end-fire array (pencil beam) and (c) An intermediate array 

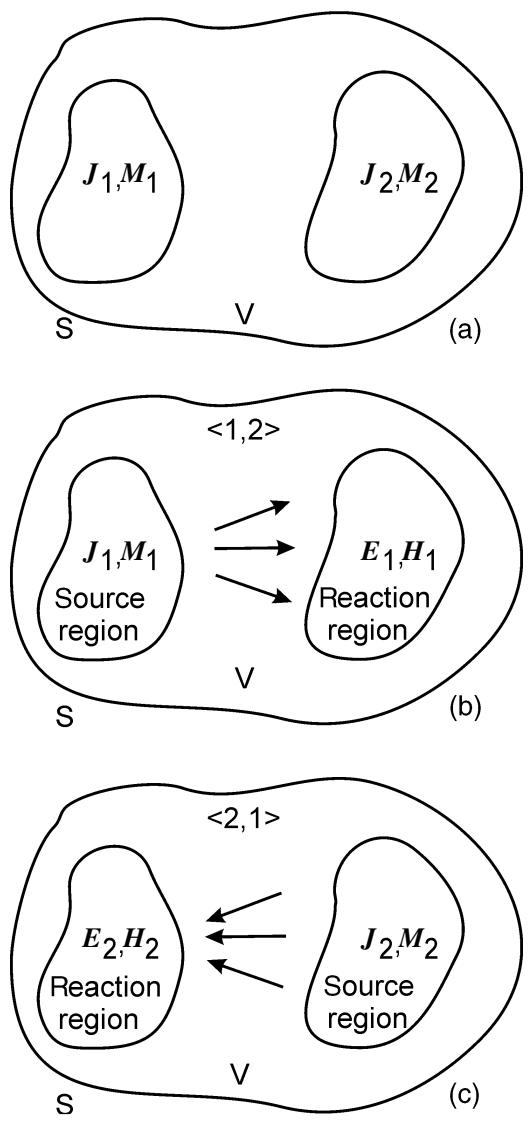

Figure 1.6 (a) Geometry of two reacted sources (b) Reaction $\langle 1,2\rangle$ and (c) Reaction $\langle 2,1\rangle$

theorem is represented with the help of Maxwell's equations and is called the 'Lorentz reciprocity theorem'. Application of the above theorem to open (unbounded) problems is of importance to antennas. Without missing the point of this book, it would be useful to give some more details.

Two pairs of sources, $\left(\boldsymbol{J}_{1}, \boldsymbol{M}_{1}\right)$ and $\left(\boldsymbol{J}_{2}, \boldsymbol{M}_{2}\right)$, existing in a volume $V$ bounded by the surface $S$ (see Fig. 1.6a), associated correspondingly with the fields $\left(\boldsymbol{E}_{1}, \boldsymbol{H}_{1}\right)$ and $\left(\boldsymbol{E}_{2}, \boldsymbol{H}_{2}\right)$, follow the reciprocity theorem, which, for $\mathrm{S}$ of infinite radius, is expressed by

$$
\iiint_{V}\left(\boldsymbol{E}_{1} \cdot \boldsymbol{J}_{2}-\boldsymbol{H}_{1} \cdot \boldsymbol{M}_{2}\right) d V=\iiint_{V}\left(\boldsymbol{E}_{2} \cdot \boldsymbol{J}_{1}-\boldsymbol{H}_{2} \cdot \boldsymbol{M}_{1}\right) d V
$$

Each of the integrals is interpreted as a coupling between the fields of a set of sources and another set of sources, which generate another set of fields. The quantity (see Fig. 1.6b)

$$
\langle 1,2\rangle=\iiint_{V}\left(\boldsymbol{E}_{1} \cdot \boldsymbol{J}_{2}-\boldsymbol{H}_{1} \cdot \boldsymbol{M}_{2}\right) d V
$$


is called a 'reaction of the field $\left(\boldsymbol{E}_{1}, \boldsymbol{H}_{1}\right)$ to the sources $\left(\boldsymbol{J}_{2}, \boldsymbol{M}_{2}\right)$ ' [28]. In a similar way, the quantity (see Fig. 1.6c)

$$
\langle 2,1\rangle=\iiint_{V}\left(\boldsymbol{E}_{2} \cdot \boldsymbol{J}_{1}-\boldsymbol{H}_{2} \cdot \boldsymbol{M}_{1}\right) d V
$$

is called a 'reaction of the field $\left(\boldsymbol{E}_{2}, \boldsymbol{H}_{2}\right)$ to the sources $\left(\boldsymbol{J}_{1}, \boldsymbol{M}_{1}\right)$ '. From (1-26)-(1-28), one can write that

$$
\langle 1,2\rangle=\langle 2,1\rangle
$$

In electromagnetics, the quantity to be measured is the field due to one source and the branch of the network is the volume containing the other source. From (1-29), it is obvious that reaction is unchanged by the interchange of source and measurement locations.

Besides the radiation pattern, another useful diagram in antennas is the polarization pattern. The polarization of an antenna is the polarization of the wave transmitted by the antenna. Polarization corresponds to a certain direction. If the direction is not stated, then it is assumed that it corresponds to the direction of the maximum. Polarization is characterized by the curve traced by the end point of the arrow representing the instantaneous electric field. The field is observed in the direction of propagation. Polarization is classified as 'linear', 'circular' or 'elliptical'. The polarization pattern of an antenna in a certain direction is defined as the angular response of a test probe rotated around the axis of the given direction of the antenna under test. In Fig. 1.7, the polarization measuring system is presented while, in Fig. 1.8, the pattern for linear, circular and elliptical polarization is given. At the radiation sphere around an antenna, polarization contains a pair of two orthogonal components. One of the components is 'co-polarization' and the other is 'cross polarization'. To accomplish the components, co-polarization is first specified at each point on the radiation sphere. After this, cross polarization is specified. For linearly polarized antennas, the orientation of the co-polar electric field in the maximum of the main beam is first specified. This maximum is directed along the polar axis of the radiation sphere. In the general case of an antenna with elliptical polarization, the major axes of the ellipse are defined. The polarization can be displayed on the surface of the Poincare sphere [29, 30]. The details of the sphere and the appropriate procedures are given in detail in the literature [31].

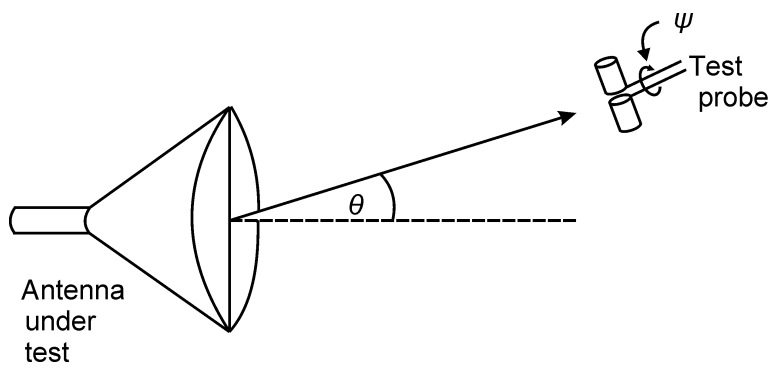

Figure 1.7 Polarization measuring system 


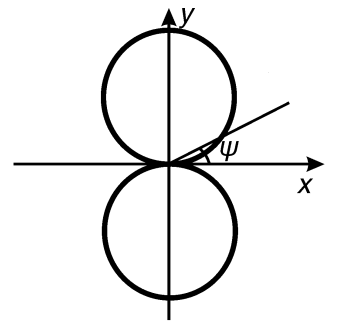

(a)

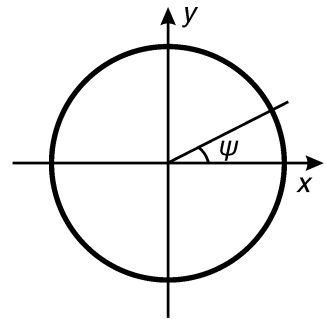

(b)

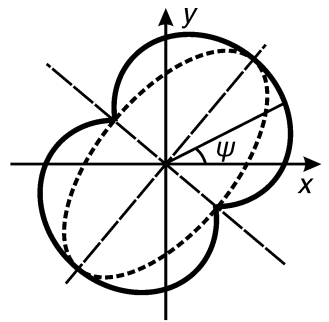

(c)

Figure 1.8 Polarization pattern (a) Linear, (b) Circular, (c) Elliptical

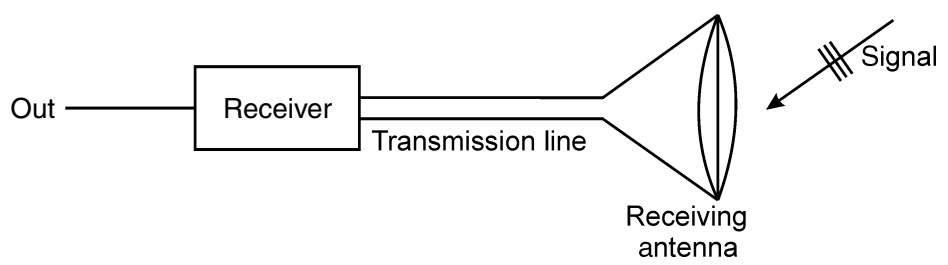

Figure 1.9 Receiving antenna with the impinged wave

\subsubsection{Antenna Effective Aperture}

The receiving antennas are mainly associated with their capturing characteristics. If a wave impinges on a receiving antenna (see Fig. 1.9) from a direction $(\theta, \phi)$, its ability to capture power can be derived by a number of equivalent areas [6].

The effective aperture (area) is one of the most common quantities. It is

$$
A_{e}=\frac{\text { Power available at the receiving antenna terminals }}{\text { Power density of incident wave }}
$$

It is assumed that the wave polarization is matched to the antenna.

Under matching conditions, the effective aperture becomes maximum and is symbolized by $A_{e m}$. The maximum effective area is related to the maximum directivity $D_{o}$ of the antenna. It is

$$
A_{e m}=\frac{\lambda^{2}}{4 \pi} D_{o}
$$

\subsubsection{Beam Efficiency}

Beam efficiency (BE) is a dimensionless quantity of an antenna related to the main beam characteristics. BE is defined as

$$
\mathrm{BE}=\frac{\text { Power transmitted (received) within the cone angle } \theta_{h}}{\text { Power transmitted (received) by the antenna }}
$$

If the main-beam maximum is directed along the $\mathrm{z}$-axis and $\theta_{h}$ is the cone angle, then (see Fig. 1.10)

$$
\mathrm{BE}=\frac{\int_{0}^{2 \pi} \int_{0}^{\theta_{h}} U(\theta, \phi) \sin \theta d \theta d \phi}{\int_{0}^{2 \pi} \int_{0}^{\pi} U(\theta, \phi) \sin \theta d \theta d \phi}
$$

Usually, the angle $\theta_{h}$ is chosen at the first null or minimum of the main beam. 


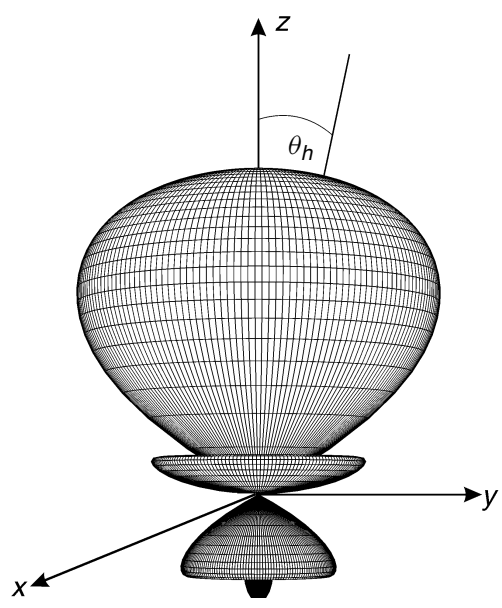

Figure 1.10 The cone angle $\theta_{h}$, where a fraction of the total radiation power of an antenna exists

Besides BE, the main-beam radiation efficiency $(n)$ is used for antenna arrays. It is a quantity useful for measuring the radiation intensity in the maximum of the main beam versus the total power of the current excitation of the elements of an array. For an array with $N$ elements, $n$ is expressed as

$$
n=\frac{\left|\sum_{i=1}^{N} I_{i}\right|^{2}}{N \sum_{i=1}^{N}\left|I_{i}\right|^{2}}
$$

In [32], it has been shown that the maximum value $n=1$ is possible for uniform cophasal excitation.

\subsubsection{Signal-to-Noise Ratio}

The signal-to-noise ratio (SNR) is a dimensionless quantity related to receiving antennas, which is defined as

$$
\mathrm{SNR}=\frac{\text { received power of the desired signal }}{\left.\frac{1}{4 \pi} \text { (noise-plus-interference power }\right)}
$$

SNR is related to the antenna gain $G(\theta, \phi)$ and the noise distribution. For uniform noise distribution, SNR is proportional to the gain.

\subsubsection{Quality Factor}

Antenna performance can be evaluated in various ways. Some figures of merit that are used for antennas include quantities such as effective aperture, gain, bandwidth, efficiency, noise power and quality factor (QF). The above are interrelated and cannot be independently optimized. 
The quality factor, represented by the symbol $Q$, is an index that measures antenna efficiency. It is defined as

$$
Q=\frac{\omega \cdot \text { average stored energy }}{\text { average power dissipated }}
$$

The above definition is the same one used in electric circuits. In an antenna, there are radiation, conduction, dielectric and surface wave losses. The quality factor contains all these losses and is written as [33],

$$
\frac{1}{Q_{t}}=\frac{1}{Q_{r}}+\frac{1}{Q_{c}}+\frac{1}{Q_{d}}+\frac{1}{Q_{s w}}
$$

$Q_{t}$ is the total $\mathrm{QF}, Q_{r}$ is the $\mathrm{QF}$ due to radiation, $Q_{c}$ is the $\mathrm{QF}$ due to conduction losses, $Q_{d}$ is the $\mathrm{QF}$ due to dielectric losses and $Q_{s w}$ is the QF due to surface waves.

For a capacitive antenna, $Q_{t}$ is $1 / \omega R C$, where $R$ includes radiation and loss resistance.

When $Q_{t}$ is large, the quality factor can be interpreted as the reciprocal of the frequency bandwidth of the antenna. Thus,

$$
Q=\frac{f_{o}}{\Delta f}
$$

As in circuit theory, $f_{o}$ is the resonant frequency and $\Delta f$ is the bandwidth for which the resistance is equal to the reactance.

In some cases, the ratio $G / Q_{t}$ is used as a figure of merit. This is known as a gain bandwidth product owing to the above-mentioned reciprocity.

For an antenna array, the so-called array $Q$ factor is given by

$$
Q=\frac{\text { sum of the excitation current magnitude squared }}{\frac{1}{4 \pi} \text { (power input to the array) }}
$$

The above expression comes from the usual meaning of the definition given in the beginning of 1.4.10. (1-21), (1-34) and (1-39) are combined for an $N$ element array and give

$$
G_{o_{a b s}}=N n Q
$$

\subsubsection{Sensitivity Factor}

An important index useful for the practical implementation of antenna arrays is the sensitivity factor K. This factor is given by [34],

$$
K=\frac{\text { sum of the excitation magnitude squared }}{\text { square of the magnitude of the array factor at } \xi_{n}=0}
$$

An ideal array is different from a real one. When an array is built, the pattern is not precisely realized to the ideal pattern. The difference comes from the currents and the location of the elements, which may differ from the desired ones.

The $K$ factor measures the susceptibility of the pattern to random errors in the position and/or the excitation of the elements of the array. 


\subsection{Antenna Input Impedance}

Antenna 'input impedance' $\left(Z_{i n}\right)$ is the impedance of an antenna at its terminals. Alternatively, $Z_{i n}$ is the ratio of the voltage (electric field) to the current (magnetic field) at a pair of terminals (at a point):

$$
Z_{\text {in }}=R_{\text {in }}+j X_{\text {in }}
$$

$R_{i n}$ and $X_{i n}$ are the input resistance and reactance of the antenna. The resistance is written as

$$
R_{i n}=R_{r}+R_{L}
$$

$R_{r}$ and $R_{L}$ are the radiation and the loss resistance of the antenna.

The antenna impedance can be derived by numerical methods and can be measured by well-tested techniques. It is noticed that the impedance is critical to the feed network design.

\subsection{Antenna Arrays Classification}

It is difficult for a single-element antenna to achieve narrow beams, low SLL, high directivity, high efficiency, null constraints, and so on due to its limited performance. A group of antenna elements, called an 'antenna array', is expected to attain the above desired performance. The types of elements, the geometry and the excitation characterize an antenna array. Knowledge of the mutual impedance between any pair of elements of the array, and the impedance of each one of them help to realize the feed network of the antenna. The network must be fairly simple to build and its size should occupy minimum space.

For simplicity in design and fabrication, the elements of an array are chosen in such a way so as to be identical and parallel. They must also be of lightweight at a relatively low cost. To meet certain performance requirements of the array, the physical space, the excitation distribution as well as the orientation and the type of elements must be derived. It is noticed that nowadays one can find a plethora of arrays. These range from complex electronically steered arrays to simpler static linear ones. A grouping of the usual antenna arrays depending on the form of their radiation pattern, their geometry, their type of elements and their excitation/fabrication is given below:

Radiation pattern: broadside, end-fire, intermediate, omnidirectional, scanned, shaped beam, multiple beam, low SLL, adaptive, null constrained.

Geometry: linear, planar, circular, flat, three dimensional, conformal.

Elements: dipoles, monopoles, loops, slots, microstrip patches, horns, spiral, helices, log periodic, monolithic, active, electrically small.

Excitation/fabrication: uniform, binomial, Chebyshev, Fourier, Franklin, HansenWoodyard, even/odd mode, series fed, taper, phased, parasitic, magnetically scanned, signal processing, superconducting, monolithic, digital beam forming.

\subsection{Array Factor Classification}

An arbitrary array with $N$ elements produces a radiation pattern that, in general, can be characterized by the superimposition of the electric fields of its elements. From (1-3) and 
(1-6), the electric field of the array is given by

$$
\boldsymbol{E}(r)=-j \omega \mu \frac{e^{-j \beta r}}{4 \pi r} \sum_{n=1}^{N} I_{n} \boldsymbol{f}_{n}(\theta, \phi) e^{j \beta r_{n} \cos \xi_{n}}
$$

$f_{n}(\theta, \phi)$ is the pattern function (vector) of the $n$th element.

As stated, the expression (1-44) contains the element patterns in the presence of the whole array. Obviously, the patterns for each element of the array are different. Even if the elements are identical and parallel, there must be some difference, which increases towards the array edge. However, as it was mentioned in 1.2, all the patterns of the elements of such an array are supposed to be approximately the same. In other cases, the radiation pattern is represented by the 'vector array pattern' and the corresponding array factor will be

$$
\boldsymbol{A F}(\theta, \phi)=\sum_{n=1}^{N} I_{n} \boldsymbol{f}_{n}(\theta, \phi) e^{j \beta r_{n} \cos \xi_{n}}
$$

The above expression derives the vector array factor, which cannot be directly related to the array factor of isotropic sources. It is noticed that the synthesis of a general array represented in (1-45) is different than the corresponding one with a scalar factor.

\section{References}

[1] S.A. Schelkunoff and H.T. Friis, Antenna Theory and Practice, John Wiley \& Sons, New York, 1952.

[2] R.W.P. King, The Theory of Linear Antennas, Harvard University Press, Cambridge, MA, 1956.

[3] J.D. Kraus, Antennas, McGraw-Hill Book Company, New York, 1988.

[4] R.C. Hansen (Ed.), Microwave Scanning Antennas, Academic Press, Vol. I, 1964, Vols. II \& III, 1966, Peninsula Publishing, New York, 1985.

[5] R.S. Elliot, Antenna Theory and Design, Prentice Hall, Englewood Cliffs, NJ, 1981.

[6] C.A. Balanis, Antenna Theory, Analysis and Design, 3rd ed., John Wiley \& Sons, New York, 2005.

[7] T. Milligan, Modern Antenna Design, McGraw-Hill Book Company, New York, 1985.

[8] N. Amitay, V. Galindo and C.P. Wu, Theory and Analysis of Phased Arrays, Wiley-Interscience, New York, 1972.

[9] M.T. Ma, Theory and Applications of Antenna Arrays, Wiley-Interscience, New York, 1974.

[10] A.W. Rudge, K. Milne, A.D. Olver and P. Knight (Eds.), The Handbook of Antenna Design, IEE/Peter Peregrinus Limited, London, 1983.

[11] Y.T. Lo and S.W. Lee, Antenna Handbook, Van Nostrad Reinhold, New York, 1988.

[12] R.C. Johnson and H. Jasik, Antenna Engineering Handbook, McGraw-Hill Book Company, New York, 1993.

[13] R.C. Mailloux, Phased Array Antenna Handbook, Artech House, Norwood, MA, 1994.

[14] J.R. James and P.S. Hall (Eds.), Handbook of Microstrip Antennas, Vol. I \& II, IEE/Peter Peregrinus Limited, London, 1989.

[15] N. Fourikis, Phased Array-Based Systems and Applications, Wiley-Interscience, New York, 1997.

[16] R.C. Hansen, Phased Array Antennas, Wiley-Interscience, New York, 1998.

[17] R.T. Compton Jr., Adaptive Antennas, Prentice Hall, Englewood Cliffs, NJ, 1988.

[18] T.S. Rappaport (Ed.), Smart Antennas, IEEE Press, Piscataway, NJ, 1998.

[19] G.V. Tsoulos (Ed.), Adaptive Antennas for Wireless Communications, IEEE Press, Piscataway, NJ, 2001.

[20] Y. Rahmat-Samii and E. Michielssen, Electromagnetic Optimization by Genetic Algorithms, WileyInterscience, New York, 1999.

[21] B.D. Popovic, M.B. Dragovic and A.R. Djordjevic, Analysis and Synthesis of Wire Antennas, Research Studies Press, John Wiley \& Sons, New York, 1982.

[22] W.L. Stutzman and G.A. Thiele, Antenna Theory and Design, John Wiley \& Sons, New York, 1998. 
[23] C.A. Balanis, Advanced Engineering Electromagnetics, John Wiley \& Sons, New York, 1989.

[24] B.D. Popovic and B.M. Kolundzija, Analysis of Metallic Antennas and Scatterers, IEE Electromagnetic Waves Series IEE, Vol. 38, London, 1994.

[25] A. Kumar, Antenna Design with Fiber Optics, Artech House, Norwood, MA, 1996.

[26] L.C. Godara, Handbook of Antennas in Wireless Communications, CRC Press, New York, 2002.

[27] J.D. Irwin, Basic Engineering Circuit Analysis, 2nd ed., Chap. 15, Macmillan, New York, 1987.

[28] V.H. Rumsey, "The Reaction Concept in Electromagnetic Theory," Phys. Rev., Series 2, Vol. 94, No. 6, pp. 1483-1491, June 15, 1954.

[29] H.G. Booker, V.H. Rumsey, G.A. Deschamps, M.I. Kales and J.I. Bonhert, "Techniques for Handling Elliptically Polarized Waves with Special Reference to Antennas," Proc. IRE, Vol. 39, pp. 533-552, May 1951.

[30] W.H. Kummer and E.S. Gillespie, “Antenna Measurements-1978," Proc. IEEE, Vol. 66, No. 4, pp. 483-507, April 1978.

[31] W.L. Stutzman, Polarization in Electromagnetic Systems, Artech House, Boston, MA, 1993.

[32] D.K. Cheng and F.I. Tseng, "Elliptical Arrays," Proc. IEEE, Vol. 114, pp. 589-594, May 1967.

[33] K.R. Carver and J.W. Mink, "Microstrip Antenna Technology," IEEE Trans. Antennas Propag., Vol. AP29, No. 1, pp. 2-24, January 1981.

[34] E.N. Gilbert and S.P. Morgan, "Optimum Design of Directive Antenna Arrays Subject to Random Variations,” Bell Syst. Tech. J., Vol. 34, pp. 637-663, May 1955. 
